\title{
Altered placental development and intrauterine growth restriction in IGF binding protein-1 transgenic mice
}

\author{
Paul A. Crossey, Claire C. Pillai, and John P. Miell \\ Department of Diabetes, Endocrinology and Internal Medicine, Guy's, King's and St. Thomas' School of Medicine, \\ London, United Kingdom
}

\begin{abstract}
IGF binding protein-1 (IGFBP-1) is a secretory product of decidualized endometrium and a major constituent of amniotic fluid. It is thought to modulate the actions of the IGFs on trophoblast cells and is therefore potentially important in regulating placental development and fetal growth. To investigate this hypothesis, we have studied the effects of decidual IGFBP-1 excess on fetoplacental growth in transgenic mice overexpressing human IGFBP-1. Endogenous fetal IGFBP-1 overexpression is associated with a transient impairment of fetal growth in midgestation. Maternal decidual IGFBP-1 excess is also associated with impaired fetal growth in midgestation independent of fetal genotype, indicating placental insufficiency. Our data also demonstrate that amniotic fluid IGFBP-1 is derived almost exclusively from maternal sources. Decidual IGFBP-1 overexpression has a marked effect on placental development. Placental morphology is abnormal in transgenic females due to altered trophoblast invasion and differentiation. These changes result in an increase in placental mass throughout pregnancy. This study provides the first compelling in vivo evidence that IGFBP-1 plays a role in placentation and suggests that IGFBP-1 has a pathological role in preeclampsia, a disorder characterized by shallow uterine invasion and altered placental development.
\end{abstract}

J. Clin. Invest. 110:411-418 (2002). doi:10.1172/JCI200210077.

\section{Introduction}

IGF-I and IGF-II play important roles in regulating the growth of embryonic and extraembryonic tissues during pregnancy. Deficiency of either IGF-I or IGF-II is associated with severe intrauterine growth failure such that birth weight is reduced by $40 \%$ (1). Deficiency of these growth factors has divergent effects on placental growth. In IGF-I knockout mice, placental growth is normal, but in IGF-II knockout mice it is impaired and may contribute in part to fetal growth restriction. Disruption of the IGF-II receptor, with an associated reduction in IGF-II clearance, leads to placental hypertrophy, further emphasizing the important role of this peptide in placental growth (2).

IGF-II is highly expressed by placental cytotrophoblasts and is believed to be important in autocrine/paracrine stimulation of trophoblast proliferation (3). The biological actions of the IGFs are stringently regulated, primarily through association with high-affinity IGF binding proteins (IGFBPs) that determine their bioavailability (4). IGFBP-1, which is

Received for publication April 11, 2000, and accepted in revised form June 13, 2002.

Address correspondence to: Paul Crossey, Department of Diabetes, Endocrinology and Internal Medicine, Guy's, Kings and St. Thomas' School of Medicine, Bessemer Road, London SE5 9PJ, United Kingdom. Phone: 44-171-737-4000 ext. 3256; Fax: 44-20-734-64771; E-mail: paul.crossey@kcl.ac.uk.

Conflict of interest: No conflict of interest has been declared. Nonstandard abbreviations used: IGF binding protein (IGFBP); human IGFBP (hIGFBP). secreted by endometrial stromal cells, is spatially and temporally positioned to play a role in regulating IGF activity at the fetomaternal interface. It modulates the metabolic and mitogenic actions of the IGFs on trophoblasts in vitro (5).

IGFBP-1 exists in a number of different isoforms that differ in their degree of phosphorylation. The highly phosphorylated isoform of IGFBP-1 has a 6-fold higher ligand binding affinity relative to the nonphosphorylated isoform. Phosphorylated IGFBP-1 has been shown to inhibit the metabolic actions of IGF-1 on trophoblast cells in vitro. Conversely, nonphosphorylated IGFBP-1 had the opposite effect, enhancing IGF activity (6). However, the physiological role, if any, of IGFBP-1 phosphorylation is unclear. The phosphorylation profile of IGFBP- 1 in maternal serum differs from that in the fetal circulation and amniotic fluid and changes as pregnancy progresses (7). In maternal serum, nonphosphorylated and intermediately phosphorylated isoforms of IGFBP-1 are found in addition to the highly phosphorylated isoform characteristic of nonpregnancy serum. IGFBP-1 in first-trimester amniotic fluid is mainly nonphosphorylated, but by the third trimester, the phosphorylated isoforms predominate.

Several studies have reported an inverse correlation between fetal growth and IGFBP-1 levels in the fetal circulation. In human fetuses that are small for their gestational age, IGFBP-1 levels in fetal cord serum are elevated (8). The relationship between IGFBP-1 levels in amniotic fluid and fetal growth is more contentious, and there have been conflicting reports on 


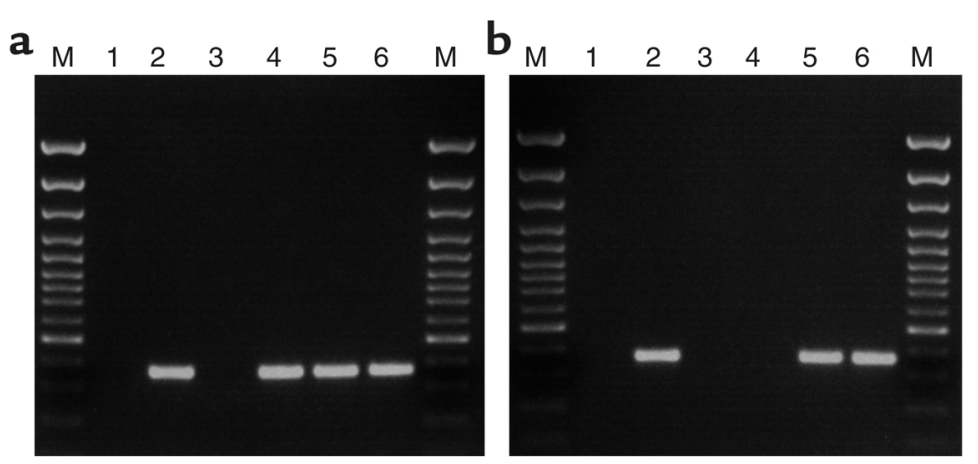

Figure 1

hIGFBP-1 gene expression in maternal decidua and fetal liver. (a) hIGFBP-1 mRNA in decidua of transgenic dams was detected by RT-PCR using a primer pair that specifically amplifies a 372-bp product from hIGFBP-1 cDNA. Lane 1, no template (negative control); lane 2, transgenic mouse liver RNA (positive control); lane 3, wild-type mouse decidua RNA (negative control); lanes 4-6, total RNA extracted decidua of transgenic females sacrificed at the indicated timepoints. M, 100-bp ladder. (b) hIGFBP-1 expression in fetal liver was detected as described in a. Lane 1, no template (negative control); lane 2, transgenic adult mouse liver RNA (positive control); lane 3, wild-type adult mouse liver RNA (negative control); lane 4, wild-type fetal liver RNA; lanes 5 and 6, liver RNA from transgenic fetuses at e 14.5 and e17.5, respectively. M, molecular size markers.

this subject $(9,10)$. IGFBP-1 levels in maternal serum are altered in preeclampsia, a disorder of pregnancy frequently associated with fetal growth restriction secondary to placental insufficiency. Serum IGFBP-1 levels are reduced in the second trimester in women who subsequently go on to develop preeclampsia, but in women with established preeclampsia, serum IGFBP-1 levels are grossly elevated $(11,12)$.

In this study, we have investigated the effects of excess IGFBP-1 on fetal and placental growth in transgenic mice overexpressing human IGFBP-1 (hIGFBP-1). Transgene expression is regulated by the native hIGFBP-1 promoter to ensure tissue-specific expression in decidual tissue and fetal liver, the major sites of IGFBP-1 expression in maternal and fetal tissues in pregnancy.

\section{Methods}

bIGFBP-1 transgenic mice. Three independent lines were established from founders born after microinjection of a human IGFBP-1 cosmid clone (13). The growth and metabolic characteristics of these lines are indistinguishable. There is evidence of sexual dimorphism in the phenotypic expression of excess IGFBP-1. Male mice exhibit marked postprandial hyperinsulinemia but have normal glucose tolerance. The metabolic and endocrine profiles of transgenic females are similar to those of sexmatched controls, as is fecundity and reproductivity. The animals used in this study were the fifth-generation backcross onto a CBA/Ca background. Parental and fetal genotypes were determined by direct PCR analysis of proteinase $\mathrm{K}$ tissue lysates using the primer combination F4221 (5'-CTAGGCTGATAATTCACTG-3') and R4457 (5'-CCGTGGCTTGGCAGGAATGTAG-3').

RNA isolation and analysis. Total RNA was extracted from frozen tissue using RNAzol B (Biogenesis Ltd.,
Poole, United Kingdom). For Northern analysis, $20 \mu \mathrm{g}$ of total RNA was electrophoresed on a denaturing formaldehyde gel, transferred to Hybond $\mathrm{N}^{+}$, and probed with mouse IGF-II full-length cDNA labeled with $\left[\alpha-{ }^{32} \mathrm{P}\right] \mathrm{dCTP}$. hIGFBP-1 gene expression was detected by reverse transcription/PCR using a primer pair that specifically amplifies the human IGFBP-1 gene (13).

Timed pregnancies. Matings between transgenic females and wild-type males $\left(F^{+} / M^{-}\right)$and the reciprocal cross, i.e., wild-type females and transgenic males $\left(F^{-} / M^{+}\right)$, were set up to obtain fetal and maternal tissues and amniotic fluid at different stages of gestation. Successful matings were indicated by the appearance of a vaginal copulation plug. Midday on the day the copulation plug was observed was considered to be 12 hours after fertilization, embryonic day 0.5 (e0.5). Pregnant females were sacrificed at e11.5, e14.5, and e17.5. Fetal anthropometric measurements were recorded, and samples of decidua, amniotic fluid, and fetal liver were collected from each conceptus. Assays for total and nonphosphorylated hIGFBP-1. Total hIGFBP-1 in amniotic fluid was measured by immunoradiometric assay (Diagnostic Systems Laboratories Inc., Webster, Texas, USA). Nonphosphorylated hIGFBP-1 was measured by an immunoradiometric assay that measures primarily the nonphosphorylated isoform of IGFBP-1 (14). Both assays show absolute specificity for human IGFBP-1.

Western ligand blotting. Samples of amniotic fluid were fractionated by electrophoresis on a $12.5 \%$ SDS polyacrylamide gel. The gel was electroblotted onto a nitrocellulose membrane (Hybond C; Amersham Biosciences, Amersham, United Kingdom). After transfer, the membrane was blocked in TBS with $0.1 \%$ Tween 20 and $1 \%$ BSA prior to incubation with $5 \times 10^{6}$ to $8 \times 10^{6}$ cpm ${ }^{125}$ I-IGF-1 for 6 hours.

bIGFBP-1 immunodetection. Western ligand membranes were stripped, blocked in TBS with $1 \%$ BSA and $0.1 \%$ Tween 20 , and incubated for two hours with a 1:1,000 dilution of a mouse anti-hIGFBP-1 monoclonal antibody (mAb 6305; Medix Biochemica, Kauniainen, Finland). Membranes were rinsed in TBS and $0.1 \%$ Tween 20 prior to and following incubation with horseradish peroxidase-conjugated anti-mouse IgG. Chemiluminescent detection was performed with ECL reagents (Amersham Biosciences) according to the manufacturer's instructions.

Placental histology. Placentas were fixed for 12-16 hours in neutral formalin, processed in an automated tissue processor, and embedded in paraffin. Paraffinembedded tissues were sectioned and mounted on slides coated with poly-L-lysine (Sigma-Aldrich, Poole, United Kingdom). The sections were stained with hematoxylin and eosin and mounted under coverslips. Placental morphometry was analyzed with the Open- 
a

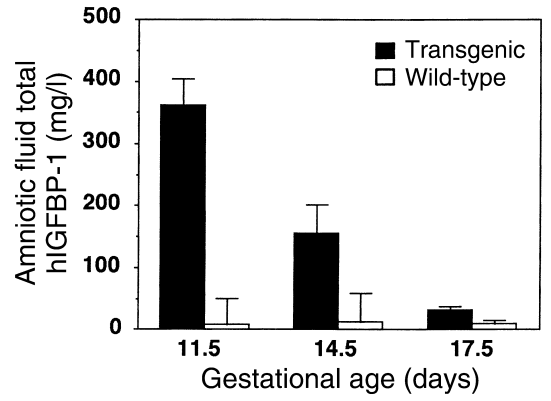

b

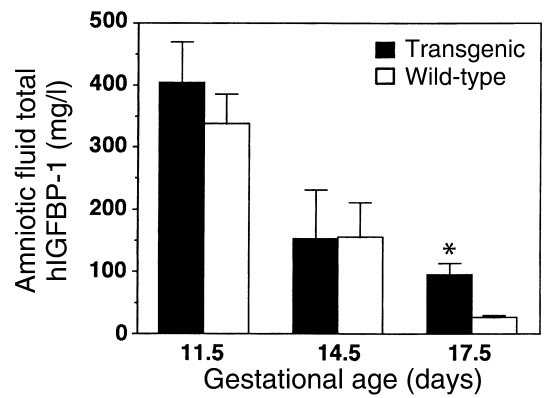

Figure 2

Amniotic fluid hIGFBP-1 levels across gestation. Total hIGFBP-1 levels in amniotic fluid were measured by a species-specific immunoradiometric assay. The data compare hIGFBP-1 levels between (a) wildtype and transgenic females and (b) wild-type and transgenic fetuses from $\mathrm{F}^{+} / \mathrm{M}^{-}$matings. Data represent mean hIGFBP-1 levels in amniotic fluid from 15-25 fetuses. Black bars, transgenic; white bars, wild-type. ${ }^{*} P<0.01$ for e17.5 transgenic versus wild-type.

lab scientific imaging software package (Improvision, Coventry, United Kingdom).

Statistical analysis. Results are presented as mean \pm SEM. Comparisons were made by ANOVA or Student $t$ test after log normalization of nonparametric data. Where $P<0.05$, ANOVA was completed using the Fisher least significant difference test.

\section{Results}

hIGFBP-1 expression in maternal and fetal tissues. hIGFBP-1 expression in decidua was detected at various gestational ages, and the results, though not strictly quantitative, indicate no gross difference in expression levels throughout gestation (Figure 1a). hIGFBP-1 mRNA was also detected in liver of transgenic fetuses at e14.5 and e17.5, but not in wild-type fetuses (Figure 1b). Technical difficulties in harvesting fetal tissues other than liver and extracting sufficient quantities of RNA from them precluded the analysis of hIGFBP-1 expression in extrahepatic tissues.

bIGFBP-1 in amniotic fluid. hIGFBP-1 was readily detectable in amniotic fluid from $F^{+} / M^{-}$crosses but was virtually undetectable in the reciprocal cross (Figure 2a). The presence of hIGFBP- 1 in amniotic fluid from $F^{+} / M^{-}$ matings was independent of fetal genotype (Figure $2 b$ ). Amniotic fluid hIGFBP-1 levels in transgenic and wildtype fetuses were similar at e11.5 and e14.5, but at e17.5 the levels were higher in transgenic fetuses $(94.0 \pm 19.3$ vs. $26.0 \pm 4.1, \mathrm{p}<0.02)$. In $F^{+} / M^{-}$matings, hIGFBP-1 lev- els in amniotic fluid declined with gestational age, falling by $80 \%$ between e 11.5 and e17.5.

Western ligand blot analysis of amniotic fluid. The IGFBP profile in amniotic fluid was investigated by Western ligand blotting. There was good agreement with the assay results, showing a decrease in the intensity of a $32-\mathrm{kDa}$ IGFBP with increasing gestational age (Figure $3 \mathrm{a})$. Western immunoblotting confirmed the identity of the 32-kDa IGFBP to be IGFBP-1 (Figure 3b). Western ligand blotting also detected a high-molecularweight IGFBP $(38-44 \mathrm{kDa})$ whose size is consistent with that of IGFBP-3.

Phosphorylation status of IIGFBP-1 in amniotic fluid. The phosphorylation profile of hIGFBP-1 in amniotic fluid from $\mathrm{F}^{+} / \mathrm{M}^{-}$matings was determined by comparing values from the total and nonphosphorylated hIGFBP-1 assays. The phosphorylated isoforms of hIGFBP-1 predominated at each timepoint (Figure 4). The proportion of nonphosphorylated hIGFBP-1 peaked at e11.5 and declined significantly by e17.5.

Effects of hIGFBP-1 overexpression on fetoplacental growth and viability. To eliminate the potential effects of litter size on fetal and placental growth, we restricted our analysis to litters of similar size (eight to ten fetuses). The effects of endogenous fetal hIGFBP- 1 overexpression on fetoplacental growth were examined by comparing growth morphometry of transgenic and wildtype fetuses from $F^{-} / M^{+}$matings (Table 1 ). There was a modest $(10 \%)$ reduction in the weight of transgenic fetuses at e11.5 compared with the wild-type, but this gap had narrowed by e14.5, and by e17.5, wild-type and transgenic fetuses had identical growth characteristics. Placental growth was similar for both wild-type and transgenic conceptuses throughout gestation.

To study the effects of decidual (i.e., maternal) hIGFBP-1 excess on fetoplacental growth, we compared the growth characteristics of wild-type fetuses in wild-type $\left(F^{-} / M^{+}\right)$and transgenic $\left(F^{+} / M^{-}\right)$dams (Table 2). Fetal growth at e 11.5 is impaired in transgenic dams. The growth restriction is independent of fetal genotype, suggesting that it is secondary to placental/decidual insufficiency. Fetal growth was similar in both uterine environments at e14.5 and e17.5.
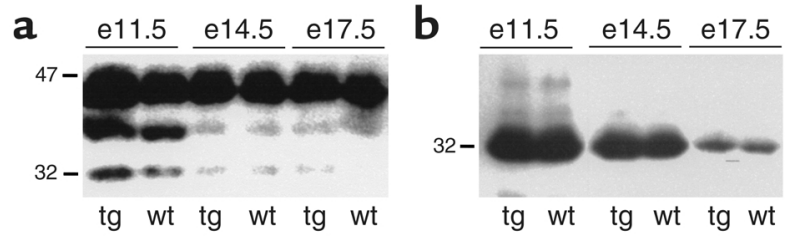

\section{Figure 3}

Western ligand and immunoblot analysis of amniotic fluid hIGFBP-1. (a) Western ligand blot of amniotic fluid from transgenic (tg) and wildtype (wt) fetuses from $\mathrm{F}^{+} / \mathrm{M}^{-}$matings. Human IGFBP-1 migrates with an apparent molecular weight of 30-32 kDa. A high-molecular-weight IGFBP (40-46 kDa) whose size is consistent with that of variantly glycosylated IGFBP-3 was also detected. (b) Western immunoblot analysis of amniotic fluid from the membrane shown in a using a mouse anti-hIGFBP-1 monoclonal antibody (mAb 6305). e, embryonic day. 


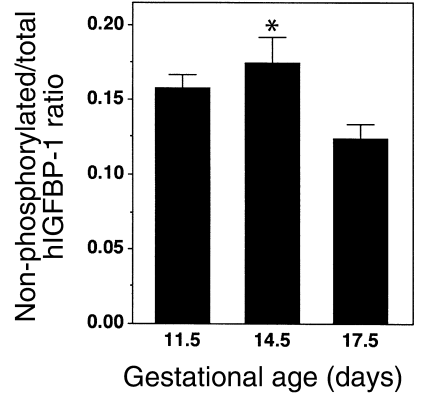

\section{Figure 4}

Phosphorylation profile of amniotic fluid hIGFBP-1. Amniotic fluid from $\mathrm{F}^{+} / \mathrm{M}^{-}$matings was assayed for total and nonphosphorylated hIGFBP-1, and the phosphorylation status of the protein was expressed as the ratio of the two values (nonphosphorylated/total hIGFBP-1). Data represent the mean of 15-25 samples of amniotic fluid. ${ }^{*} P<0.01$ for e14.5 versus e17.5.

Maternal decidual IGFBP-1 excess had a lasting effect on placental growth. Mean placental weights were greater in transgenic females at all timepoints, irrespective of fetal genotype.

Fetal viability was influenced by parental genotype. In $F^{-} / M^{+}$matings, no fetal deaths were observed in over 100 conceptuses from a total of 12 litters. In contrast, in matings involving a transgenic female, the rate of fetal death was $12 \%$.

Placental IGF-II expression. Placental IGF-II expression was investigated by Northern blot analysis using a full-length murine cDNA probe (Figure 5). No significant difference was found between expression levels in hIGFBP-1 transgenic and wild-type mice at e11.5 or e 14.5 .

Placental histology. Comparison of placental morphology and histology indicated differences in trophoblast invasion and differentiation in transgenic mice (Figure 6). The decidual component of placentas from wildtype females was greater than that of placentas from transgenic females. Placentas from $F^{+} / M^{-}$matings also had evidence of aberrant trophoblast differentiation. The junctional zone was larger in placentas from transgenic females than in placentas from wild-type females, and the ratio of spongiotrophoblasts to glycogen cells was higher in the transgenic females. The most striking difference in placental structure was the size of the labyrinthine zone. This was grossly enlarged in transgenic females, occupying approximately $80 \%$ of the cross-sectional area of the placenta compared with about $50 \%$ in placentas from wild-type females.

\section{Discussion}

In this study, we have determined the effects of endogenous fetal and maternal decidual overexpression of IGFBP-1 on placental and fetal growth in transgenic mice. A number of other transgenic models of IGFBP-1 overexpression have been developed, two of which have reported reproductive and developmental abnormalities. Gay et al. used the human $\alpha 1$-antitrypsin promoter to target IGFBP-1 expression to the liver (15), while Rajkumar and colleagues used the mouse phosphoglycerate kinase ( $p g k$ ) promoter to drive expression of the rat IGFBP-1 structural gene (16). These investigators reported reduced fecundity and litter size and increased ante and postnatal mortality. It is extremely unlikely that these effects are due to endometrial or placental abnormalities, as neither promoter is active in these tissues. In the latter model, the authors conclude that expression of the transgene in maternal tissues has no significant negative effect on implantation.

Given the limitations of these models for exploring IGFBP-1 function in placentation, we undertook the present study using transgenic mice with targeted overexpression of IGFBP-1 in the endometrium. This was achieved using the human IGFBP-1 structural gene and its native promoter as a construct for generating transgenic mice. The promoter contains all cis-acting sequences necessary for normal cell-specific and regulated gene expression. The latter include putative progesterone response elements that mediate the dramatic increase in endometrial stromal cell IGFBP-1 gene expression when they are decidualized in vitro (17). For these reasons, this model of IGFBP-1 overexpression is uniquely suited to addressing the role of IGFBP- 1 in placentation. We have previously shown that IGFBP-1 transgenic mice have subtle abnormalities of glucose homeostasis. However, this is observed only in males. Females have metabolic and endocrine profiles similar to those of sex-matched wild-type animals when measured under a variety of nutritional states. Therefore, maternal metabolic and endocrine dysfunction cannot

Table 1

Effects of endogenous fetal hIGFBP-1 overexpression: growth morphometry of wild-type and transgenic fetuses in $F^{-} / M^{+}$matings at e11.5, e14.5, and e17.5

\begin{tabular}{lcccccc}
\hline & \multicolumn{2}{c}{$\mathrm{e} 11.5$} & \multicolumn{2}{c}{$\mathrm{e} 14.5$} & & $\mathrm{e} 17.5$ \\
& $\mathrm{wt}$ & $\mathrm{tg}$ & $\mathrm{wt}$ & $\mathrm{tg}$ & $\mathrm{wt}$ & $\mathrm{tg}$ \\
Fetal weight $(\mathrm{mg})$ & $47 \pm 2$ & $42 \pm 2$ & $239 \pm 7$ & $232 \pm 6$ & $910 \pm 19$ & $916 \pm 17$ \\
Placental weight $(\mathrm{mg})$ & $41 \pm 2$ & $44 \pm 2$ & $88 \pm 5$ & $85 \pm 3$ & $99 \pm 3$ & $102 \pm 3$ \\
Crown rump length (mm) & $7.6 \pm 0.1$ & $7.5 \pm 0.1$ & $11 \pm 1.4$ & $10.9 \pm 1.5$ & $20.6 \pm 0.4$ & $19.8 \pm 0.4$ \\
Placental-to-fetal weight ratio & $0.9 \pm 0.1$ & $1.1 \pm 0.1^{\mathrm{A}}$ & $0.4 \pm 0.02$ & $0.4 \pm 0.01$ & $0.1 \pm 0.01$ & $0.1 \pm 0.003$
\end{tabular}

Data are presented as mean \pm SEM for 14-20 wild-type and transgenic fetuses. The placental-to-fetal weight ratio allows an instant comparison of both placental and fetal weight in wild-type and transgenic fetuses. ${ }^{A} P<0.05$ for transgenic versus wild-type fetuses. 
Table 2

Effects of decidual hIGFBP-1 overexpression: fetoplacental growth in transgenic versus wild-type dams

\begin{tabular}{|c|c|c|c|c|c|c|c|}
\hline \multirow{2}{*}{$\begin{array}{l}\text { Growth } \\
\text { parameter }\end{array}$} & \multirow{2}{*}{$\begin{array}{l}\text { Maternal } \\
\text { genotype }\end{array}$} & \multicolumn{6}{|c|}{ Fetal genotype } \\
\hline & & wt & $\operatorname{tg}$ & wt & $\operatorname{tg}$ & wt & $\operatorname{tg}$ \\
\hline & & \multicolumn{2}{|c|}{ e11.5 } & \multicolumn{2}{|c|}{ e14.5 } & \multicolumn{2}{|c|}{ e17.5 } \\
\hline Fetal weight (mg) & $\begin{array}{l}\text { wt } \\
\text { tg }\end{array}$ & $\begin{array}{l}47 \pm 2^{A} \\
41 \pm 2\end{array}$ & $\begin{array}{l}42 \pm 2^{A} \\
36 \pm 2\end{array}$ & $\begin{array}{l}239 \pm 7 \\
237 \pm 6\end{array}$ & $\begin{array}{l}232 \pm 6 \\
221 \pm 17\end{array}$ & $\begin{array}{l}910 \pm 19 \\
901 \pm 41\end{array}$ & $\begin{array}{l}916 \pm 17 \\
894 \pm 58\end{array}$ \\
\hline Placental weight (mg) & $\begin{array}{l}\text { wt } \\
\text { tg }\end{array}$ & $\begin{array}{l}41 \pm 2^{A} \\
52 \pm 4\end{array}$ & $\begin{array}{l}44 \pm 4^{B} \\
53 \pm 4\end{array}$ & $\begin{aligned} 88 & \pm 5^{A} \\
101 & \pm 4\end{aligned}$ & $\begin{aligned} 85 & \pm 3^{B} \\
103 & \pm 6\end{aligned}$ & $\begin{aligned} 99 & \pm 3^{A} \\
114 & \pm 6\end{aligned}$ & $\begin{array}{l}102 \pm 3 \\
109 \pm 3\end{array}$ \\
\hline Crown rump length (mm) & $\begin{array}{l}\text { wt } \\
\text { tg }\end{array}$ & $\begin{array}{l}7.6 \pm 0.1 \\
7.8 \pm 0.2\end{array}$ & $\begin{array}{l}7.5 \pm 0.1 \\
7.7 \pm 0.2\end{array}$ & $\begin{array}{r}11 \pm 1.4 \\
10.9 \pm 1.1\end{array}$ & $\begin{array}{l}10.9 \pm 1.5 \\
10.7 \pm 0.9\end{array}$ & $\begin{array}{l}19.8 \pm 0.4 \\
19.5 \pm 0.5\end{array}$ & $\begin{array}{l}20.6 \pm 0.4 \\
19.6 \pm 0.5\end{array}$ \\
\hline Placental-to-fetal weight ratio & $\begin{array}{l}\text { wt } \\
\text { tg }\end{array}$ & $\begin{array}{l}0.9 \pm 0.1^{\mathrm{A}} \\
1.2 \pm 0.1\end{array}$ & $\begin{array}{l}1.1 \pm 0.1^{c} \\
1.5 \pm 0.1\end{array}$ & $\begin{array}{l}0.4 \pm 0.02^{B} \\
0.4 \pm 0.02\end{array}$ & $\begin{array}{l}0.4 \pm 0.01^{\mathrm{D}} \\
0.4 \pm 0.02\end{array}$ & $\begin{array}{l}0.1 \pm 0.01^{\mathrm{A}} \\
0.1 \pm 0.01\end{array}$ & $\begin{array}{l}0.1 \pm 0.003 \mathrm{D} \\
0.1 \pm 0.01\end{array}$ \\
\hline
\end{tabular}

Data are presented as mean \pm SEM for 14-20 wild-type fetuses or 12-17 transgenic fetuses (wild-type or transgenic indicates maternal and fetal genotype) The placental-to-fetal weight ratio allows an instant comparison of both placental and fetal weight in wild-type and transgenic fetuses. ${ }^{A} P<0.05$, ${ }^{B} P<0.01$, ${ }^{c} P<0.005$ for differences between fetal growth parameters in wild-type versus transgenic dams; ${ }^{D}$ nonsignificant trend, $P<0.1$

be implicated in any possible abnormalities of fetoplacental growth and development.

Our finding that hIGFBP-1 can be detected in amniotic fluid only from matings involving a transgenic female is a highly significant observation. It provides definitive in vivo evidence that IGFBP-1 is derived solely from maternal decidua. Earlier studies had merely inferred this by correlating IGFBP-1 synthesis rates of decidual explants in culture with levels of the protein in amniotic fluid (18). The observation that hIGFBP-1 levels in amniotic fluid are not related to fetal genotype is consistent with our findings, since all fetuses, irrespective of their genotype, grow in intimate contact with the maternal decidua. hIGFBP-1 levels in amniotic fluid tend to be slightly higher in transgenic compared with wild-type fetuses at e17.5, suggesting that some IGFBP-1 may be derived from endogenous fetal production. In the reciprocal mating, hIGFBP-1 levels in amniotic fluid are virtually undetectable, proving that the fetal contribution to amniotic fluid IGFBP-1 levels is negligible.

hIGFBP-1 levels in amniotic fluid from $\mathrm{F}^{+} / \mathrm{M}^{-}$matings peak at e11.5 and decline thereafter. In human subjects, amniotic fluid IGFBP-1 levels rise slowly until about 10 weeks of gestation. Between 10 and 16 weeks, levels increase dramatically before declining gradually to term. Amniotic fluid IGFBP-1 levels are determined by the rates of decidual synthesis and diffusion across the extraembryonic membranes (amnion and chorion). IGFBP-1 synthesis increases dramatically in the first trimester, due largely to the effects of progesterone on endometrial stromal cell IGFBP-1 gene expression.
Cytokines such as IL- $1 \beta$ may also play a role in regulating IGFBP-1 synthesis (19). The amnion and the chorion are semipermeable and limit passage of IGFBP-1 into the amniotic sac. These membranes fuse between 10 weeks and 16 weeks, facilitating rapid diffusion of IGFBP-1 into the amniotic sac.

Amniotic fluid from IGFBP-1 transgenic females also contains a high-molecular-weight IGFBP (40-46 $\mathrm{kDa}$ ) whose size is consistent with that of glycosylated IGFBP-3. IGFBP-3 levels in amniotic fluid from wild-type and transgenic fetuses are similar throughout gestation. There is no evidence to suggest a compensatory downregulation of IGFBP-3 to normalize total IGFBP levels in amniotic fluid. Compensatory changes in IGFBP levels have been reported in IGFBP-2-deficient mice, but not in other IGFBP-1 transgenic models $(20,21)$.

We have used commercially available immunoassays to measure levels of hIGFBP-1 and to determine its phosphorylation status in amniotic fluid from transgenic females. We previously showed that estimates of IGFBP-1 phosphorylation status derived from these assays correlate precisely with values obtained by ligand blotting after PAGE in the presence of the nonionic detergent $n$-octyl glucoside (22). The ratio of nonphosphorylated to total hIGFBP-1 in amniotic fluid from transgenic dams declines significantly between e 14.5 and e17.5, indicating increased protein phosphorylation. Similar changes in the phosphorylation status of amniotic fluid IGFBP-1 have been described in human studies (6).

\section{Figure 5}

Northern blot analysis of placental IGF-II expression. Total RNA from e11.5 and e14.5 placentas from wild-type $\left(\mathrm{F}^{-} / \mathrm{M}^{+}\right)$and transgenic $\left(\mathrm{F}^{+} / \mathrm{M}^{-}\right)$females was probed with the murine IGF-II full-length cDNA. The membranes were stripped and reprobed with murine GAPDH cDNA to verify equal sample loading.
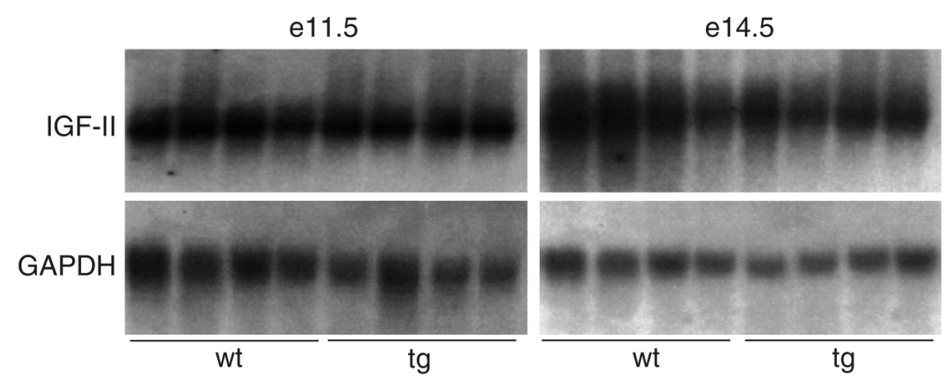
a

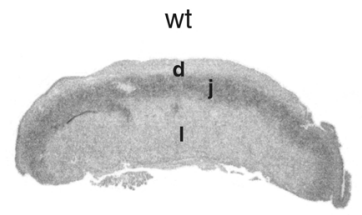

b

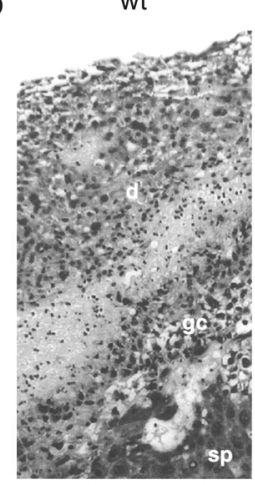

C

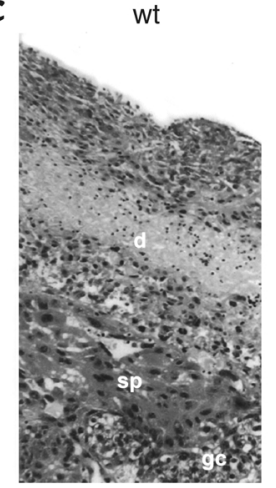

$\operatorname{tg}$

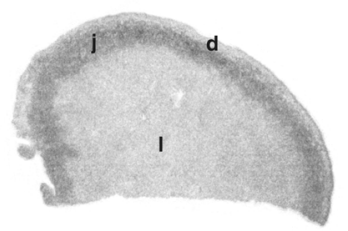

wt

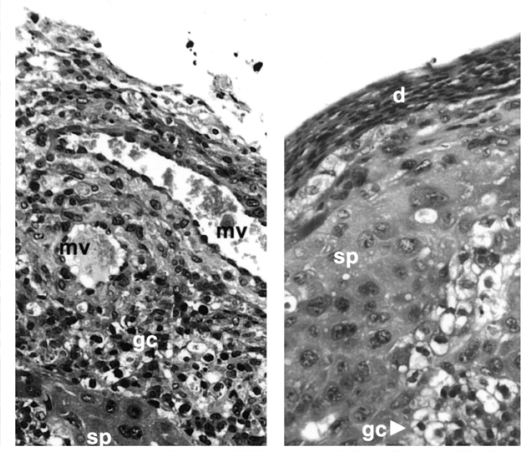

$\operatorname{tg}$

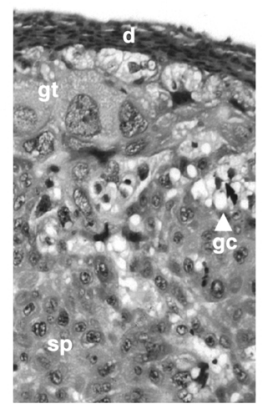

Our results demonstrate that endogenous $\left(F^{-} / M^{+}\right)$fetal hIGFBP-1 overexpression has a modest inhibitory effect on fetal growth. This is consistent with data from the pgk-rIGFBP-1 transgenic mice developed by Rajkumar et al. In human and animal studies of uteroplacental insufficiency, IGFBP-1 levels in the fetal circulation are elevated (23). In uteroplacental insufficiency, there is a reduction in placental perfusion and substrate delivery to the fetus, which is, in effect, starved. In these circumstances, IGFBP-1 levels increase as they would in adult life. It has been postulated that increased IGFBP-1 levels play a role in fetal growth restriction.

IGFBP-1 overexpression has a modest and transient effect on fetal growth in contrast with that associated with deficiency of either IGF-I or IGF-II. This is to be expected, since there is complete loss of IGF function in the latter. The onset of growth restriction in IGF-Ior IGF-II-deficient mice is first apparent between e11 and $\mathrm{e} 13.5$, as it is in IGFBP-1 transgenic fetuses. However, increased circulating levels of IGFBP-1 are not sufficient to exert a sustained inhibitory effect on fetal growth. Absolute levels of IGFBP-1 may be less important than the ratios of the phosphorylated and nonphosphorylated isoforms of the protein. Thus, while

\section{Figure 6}

Placental histology in hIGFBP-1 transgenic mice. (a) Gross morphology. The different functional layers of the mouse placenta are shown ( $d$, decidual; j, junctional; I, labyrinthine). Differences in placental cross-sectional area and the relative proportions of the different functional layers of the placenta are clearly evident. (b) Decidual thickness. The thickness of the decidual layer is greater in placentas from wild-type females, indicating reduced trophoblast invasion in transgenic females. The labyrinthine zone is grossly enlarged, occupying approximately $80 \%$ of the cross-sectional area of the placenta compared with about $50 \%$ in wild-type placentas. sp, spongiotrophoblast; mv, maternal blood vessel; gc, glycogen cells; gt, trophoblast giant cells. (c) Trophoblasts in the junctional zone. The junctional zone is larger in placentas from transgenic females. There are similar numbers of glycogen cells and spongiotrophoblasts in placentas from wild-type females. In transgenic mice, spongiotrophoblasts are the predominant cell type; glycogen cells are relatively scarce compared with placentas from wild-type females.

total levels may increase, the relative levels of the stimulatory and inhibitory isoforms of IGFBP-1 may negate each other. The phosphorylation profile of IGFBP-1 in conditioned medium of human second-trimester fetal liver explants shows that both the phosphorylated and nonphosphorylated isoforms are present (18).

Our data also show that maternal IGFBP-1 overexpression can independently influence fetal growth, suggesting that decidual IGFBP-1 excess is associated with placental insufficiency. The effects of decidual and endogenous fetal overexpression are additive, as transgenic fetuses in the $\mathrm{F}^{+} / \mathrm{M}^{-}$cross are the smallest and wildtype fetuses in the $F^{-} / M^{+}$cross are the biggest of the four possible combinations. The most striking observation associated with decidual IGFBP-1 excess is increased placental mass. The increase in placental mass cannot be attributed to changes in placental IGF-II expression. Altered IGF-II availability at the fetomaternal interface is a more plausible hypothesis. Our data indicate that the phosphorylated isoforms of hIGFBP-1 predominate at the fetomaternal interface and would therefore predict reduced IGF bioavailability.

Histological analysis of placentas provides evidence for the cellular and morphological basis of placentomegaly in hIGFBP-1 transgenic dams. Trophoblast invasion of the decidualized endometrium is less pronounced in transgenic females than in wild-type females. The depth of the decidual component of the placenta is essentially a measure of how far placental trophoblasts have penetrated the maternal decidua. There are a number of potential mechanisms by which decidual IGFBP-1 excess might impair trophoblast invasion. First, trophoblast proliferation and invasion may be attenuated as a consequence of reduced IGF bioavailability in the decidual microenvironment. Reduced IGF bioavailability may also influence trophoblast differentiation, perhaps favoring the growth of noninvasive over invasive trophoblast lineages. Alternatively, IGFBP-1 may have direct IGF-independent effects on trophoblast invasion. Such effects have been demonstrated in $\mathrm{CHO}$ cells and trophoblasts in vitro. Irwin and Giudice 
reported that IGFBP-1 inhibited trophoblast invasion of endometrial stromal cells in vitro (24). However, a subsequent study by Gleeson et al. contradicts these findings, showing increased trophoblast invasion in response to IGFBP-1 (25). In both studies, the authors show that these effects, although divergent, are mediated through binding of IGFBP- 1 to $\alpha 5 \beta 1$ integrin.

Other aspects of placental development are altered in IGFBP-1 transgenic mice. The relative sizes of the different functional zones of the placenta differ markedly from those of the wild-type, as do the proportions of various differentiated trophoblast cell types. Some aspects of placental morphology in IGFBP-1 transgenic mice are reminiscent of IGF-II-deficient mice (26). In both cases, spongiotrophoblast cells account for a greater proportion of the junctional zone. However, these models differ in that placental mass is reduced in IGF-II null mice, whereas it is increased in IGFBP-1 transgenic females. Increased placental mass in hIGFBP-1 transgenic mice is largely due to an increase in the size of the labyrinthine zone. At this time, we cannot exclude the possibility that this is a primary manifestation of decidual IGFBP-1 excess; however, although it is somewhat speculative, we feel it is more likely to be a physiological adaptation to maximize nutrient transfer to the fetus.

The placenta can undergo structural and functional adaptations in response to an unfavorable maternal milieu. Such changes have been noted in both pathological and experimental scenarios of maternal anemia, undernutrition, and hypoxia (27-29). In human placentas that develop under conditions of hypobaric hypoxia, significant changes in fetal capillary volume (increased vessel diameter and length) and villus membrane thickness have been reported (30). These adaptations function to facilitate increased gaseous exchange between the maternal and fetal circulation. Hypoxia is probably a consequence of reduced trophoblast invasion of the decidua, and therefore similar adaptations may occur in placentas of IGFBP-1 transgenic mice. The exchange functions of the rodent placenta are largely confined to the labyrinthine zone. Thus, compensatory changes in fetal vascularization could result in an increase in labyrinthine tissue volume. Such changes may account for the relatively mild fetal growth restriction that is observed, which might otherwise be more severe. These issues are the subject of ongoing studies in our laboratory.

Preeclampsia is an important clinical condition of altered placental growth and intrauterine growth restriction. Although the pathophysiology of this condition has not been fully elucidated, it has been established that the primary defect is one of shallow trophoblast invasion. Histochemical analysis of the placenta in preeclamptic pregnancies has shown increased proliferation of anchoring (noninvasive) trophoblasts and a decreased fraction of extravillus (invasive) trophoblasts (31). Interestingly, maternal serum IGFBP-1 levels are altered in preeclampsia.
Serum IGFBP-1 levels are reduced in the first trimester in women who subsequently develop preeclampsia, but in women with established disease, IGFBP-1 levels are grossly elevated. The fluctuation of circulating peptide levels is believed to reflect changes in decidual IGFBP-1 synthesis.

In conclusion, this report presents definitive in vivo evidence that IGFBP-1 in amniotic fluid is exclusively decidual in origin and demonstrates that decidual IGFBP-1 excess is associated with altered placental development and impaired fetal growth. This study provides the first compelling in vivo evidence that IGFBP-1 plays a significant role in placental growth and morphogenesis. Reduced trophoblast invasion and impaired fetal growth are characteristic features of preeclampsia, a disorder that is associated with aberrant decidual IGFBP- 1 synthesis. Therefore, our findings suggest that IGFBP-1 has a pathological role in the abnormalities of placental development that are the primary defect in preeclampsia.

\section{Acknowledgments}

This work was supported by the Wellcome Trust and by Diabetes UK.

1. Baker, J., Liu, J-P., Robertson, E.J., and Efstratiadis, A. 1993. Role of insulin-like growth factors in embryonic and postnatal growth. Cell. 75:73-82.

2. Lau, M.M., et al. 1994. Loss of the imprinted IGF2/cation-independent mannose 6-phosphate receptor results in fetal overgrowth and perinatal lethality. Genes Dev. 8:2953-2963.

3. Han, V.K.M., Bassett, N., Walton J., and Challis, J.R.G. 1996. The expression of insulin-like growth factor (IGF) and IGF-binding protein (IGFBP) genes in the human placenta and membranes: evidence for IGFIGFBP interactions at the feto-maternal interface. J. Clin. Endocrinol. Metab. 81:2680-2693.

4. Jones, J.I., and Clemmons, D.R. 1995. Insulin-like growth factors and their binding proteins: biological actions. Endocr. Rev. 16:3-34.

5. Ritvos, O., et al.1988. Insulin-like growth factor (IGF) binding protein from human decidua inhibits the binding and biological action of IGF-I in cultured choriocarcinoma cells. Endocrinology. 122:2150-2157.

6. Yu, J., Iwashita, M., Kudo, Y., and Takeda, Y. 1998. Phosphorylated insulin-like growth factor (IGF)-binding protein-1 (IGFBP-1) inhibits while non-phosphorylated IGFBP-1 stimulates IGF-1-induced amino acid uptake by cultured trophoblast cells. Growth Horm. IGF Res. 8:65-70.

7. Westwood, M., Gibson, J.M., Davies, A.J., Young, R.J., and White, A. 1994. The phosphorylation pattern of insulin-like growth factor-binding protein-1 in normal plasma is different from that in amniotic fluid and changes during pregnancy. J. Clin. Endocrinol. Metab. 79:1735-1741.

8. Langford, K., et al. 1994. The pathophysiology of the insulin-like growth factor axis in fetal growth failure: a basis for programming by undernutrition? Eur. J. Clin. Invest. 24:851-856.

9. Hakala-Ala-Pietila, T.H., Koistinen, R.A., Salonen, R.K., and Seppala, M.T. 1993. Elevated second-trimester amniotic fluid concentration of insulin-like growth factor binding protein-1 in fetal growth retardation. Am. J. Obstet. Gynecol. 169:35-39.

10. Verhaeghe, J., et al.1999. IGF-I, IGF-II, IGF binding protein 1, and C-peptide in second trimester amniotic fluid are dependent on gestational age but do not predict weight at birth. Pediatr. Res. 46:101-108.

11. Giudice, L.C., Martina, N.A., Crystal, R.A., Tazuke, S., and Druzin, M. 1997. Insulin-like growth factor binding protein-1 at the maternal-fetal interface and insulin-like growth factor-I, insulin-like growth factor-II, and insulin-like growth factor binding protein-1 in the circulation of women with severe pre-eclampsia. Am. J. Obstet. Gynecol. 176:751-758.

12. De Groot, C.J., O’Brien, T.J., and Taylor, R.N. 1996. Biochemical evidence of impaired trophoblastic invasion of decidual stroma in women destined to have pre-eclampsia. Am. J. Obstet. Gynecol. 175:24-29.

13. Crossey, P.A., Jones, J.S., and Miell, J.P. 2000. Dysregulation of the insulin/insulin like growth factor binding protein-1 axis in transgenic mice is associated with hyperinsulinemia and glucose intolerance. Diabetes. 49:457-465.

14. Khosravi, M.J., Diamandi, A., and Mistry, J. 1997. Immunoassay of 
insulin-like growth factor binding protein-1. Clin. Chem. 43:523-532.

15. Gay, E., et al. 1997. Liver-specific expression of human insulin-like growth factor binding protein-1 in transgenic mice: repercussions on reproduction, ante- and perinatal mortality and postnatal growth. Endocrinology. 138:2937-2947.

16. Huang, H., Rajkumar, K., and Murphy, L.J. 1997. Reduced fecundity in insulin-like growth factor-binding protein-1 transgenic mice. Biol. Reprod. 56:284-289.

17. Tseng, L., Gao, J., Mazella, J., Zhu, H.H., and Lane, B. 1997. Differentiation-dependent and cell-specific regulation of the hIGFBP-1 gene in human endometrium. Ann. NY Acad. Sci. 828:27-37.

18. Martina, N.A., et al. 1997. Gestational age-dependent expression of insulin-like growth factor-binding protein-1 (IGFBP-1) phosphoisoforms in human extraembryonic cavities, maternal serum, and decidua suggests decidua as the primary source of IGFBP-1 in these fluids during early pregnancy. J. Clin. Endocrinol. Metab. 82:1894-1898.

19. Strakova, Z., Srisuparp, S., and Fazleabas, A.T. 2000. Interleukin-1beta induces the expression of insulin-like growth factor binding protein-1 during decidualization in the primate. Endocrinology. 141:4664-4670.

20. Wood, T.L., Rogler, L.E., Czick, M.E., Schuller, A.G.P., and Pintar, J.E. 2000. Selective alterations in organ sizes in mice with a targeted disruption of the insulin-like growth factor binding protein-2 gene. Mol. Endocrinol. 14:1472-1482.

21. Huang, H., Rajkumar, K., and Murphy, L.J. 1997. Expression of insulinlike growth factor binding proteins (IGFBPs) in IGFBP-1 transgenic mice. J. Endocrinol. 152:99-108.

22. Miell, J.P., et al. 1997. The maternal insulin-like growth factor (IGF) and IGF-binding protein response to trisomic pregnancy during the first trimester: a possible diagnostic tool for trisomy 18 pregnancies. J. Clin. Endocrinol. Metab. 82:287-292.

23. Unterman, T.G., Simmons, R.A., Glick, R.P., and Ogata, E.S. 1993. Cir- culating levels of insulin, insulin-like growth factor-I (IGF-I), IGF- II, and IGF-binding proteins in the small for gestational age fetal rat. Endocrinology. 132:327-336.

24. Irwin, J.C., and Giudice, L.C. 1998. Insulin-like growth factor binding protein-1 binds to placental cytotrophoblast alpha5beta 1 integrin and inhibits cytotrophoblast invasion into decidualized endometrial stromal cultures. Growth Horm. IGF Res. 8:21-31.

25. Gleeson, L.M., Chakraborty, C., McKinnon, T., and Lala, P.K. 2001 Insulin-like growth factor-binding protein 1 stimulates human trophoblast migration by signaling through alpha 5 beta 1 integrin via mitogen-activated protein kinase pathway. J. Clin. Endocrinol. Metab. 86:2484-2493.

26. Lopez, M.F., Dikkes, P., Zurakowski, D., and Villa-Komaroff, L. 1996 Insulin-like growth factor II affects the appearance and glycogen content of glycogen cells in the murine placenta. Endocrinology. 137:2100-2108

27. Godfrey, K.M., Redman, C.W.G., Barker, D.J.P., and Osmond, C. 1991. The effect of maternal anaemia and iron deficiency on the ratio of fetal weight to placental weight. Br. J. Obstet. Gynaecol. 98:886-891.

28. Lumey, L.H. 1998. Compensatory placental growth after restricted maternal nutrition in early pregnancy. Placenta. 19:105-111.

29. Reshetnikova, O.S., Burton, G.J., and Milovanov, A.P. 1994. Effects of hypobaric hypoxia on the fetoplacental unit: the morphometric diffusing capacity of the villous membrane at high altitude. Am. J. Obstet. Gynecol. 171:1560-1565.

30. Burton, G.J., Reshetnikova, O.S., Milovanov, A.P., and Teleshova, O.V. 1996. Stereological evaluation of vascular adaptations in human placental villi to differing forms of hypoxic stress. Placenta. 17:49-55.

31. Redline, R.W., and Patterson, P. 1995. Pre-eclampsia is associated with an excess of proliferative immature intermediate trophoblast. Hum Pathol. 26:594-600. 